\title{
Ending weight bias and the stigma of obesity
}

\author{
A new Consensus Statement published in Nature Medicine calls for the end of weight bias and the \\ stigma of obesity. Nature Reviews Endocrinology is proud to support this initiative.
}

\section{Nature Reviews Endocrinology is proud to support the pledge and help end weight bias and obesity stigma}

Obesity and overweight have increased in prevalence in the past few decades, reaching pandemic levels in many countries. People with overweight and obesity are known to be at increased risk of a range of medical complications. In addition, it is becoming increasingly clear that people with obesity and overweight are also facing stigma in many aspects of their lives, which not only has a damaging effect on the individual's mental health but can also influence the quality of health care that affected people receive. In a Consensus Statement published in Nature Medicine, Francesco Rubino and colleagues call for an end to weight bias and obesity stigma and set out a pledge to help achieve this aim ${ }^{1}$.

Weight bias and obesity stigma are rooted in the misconception that body weight is easily controlled by making changes to the diet and physical activity levels. In reality, the factors that influence body weight are complex and include genetics, epigenetics, the environment, societal factors and medications ${ }^{2}$. Indeed, lifestyle and behavioural interventions that involve reduced calorie intake and increased physical activity levels rarely result in long-lasting changes in body weight, owing to complex hormonal and metabolic adaptations for maintaining body weight ${ }^{2}$. In short, altering body weight is not as simple as eating less and moving more.

Despite obesity being generally accepted as a chronic disease ${ }^{3}$, obesity stigma is highly prevalent and has far reaching effects ${ }^{4}$. Weight bias and obesity stigma can affect an individual's everyday life - from their salary to the likelihood that they will seek health care and the care that they then receive. In their Consensus Statement, the international panel of 36 experts, including representatives from a range of institutions and organizations, such as the World Obesity Federation and the American Diabetes Association, suggest that weight bias and obesity stigma can also have much wider negative effects, informing public health policies, limiting access to appropriate treatments and influencing the direction of research.

The Consensus Statement was developed to inform health-care professionals, policy makers and the public about the factors that have led to obesity stigma and the resulting negative effects. The authors of the Consensus Statement recognize that body weight regulation is not something an individual can easily control. Furthermore, they condemn the use of stigmatizing language, images, attitudes, policies and weight-based discrimination wherever they occur. Several recommendations have been included to help end obesity stigma, such as updating the curricula of health-care providers to ensure a more complete understanding of the causes of obesity and ensuring public health authority policies do not promote weight-based stigma.

The Consensus Statement also includes a pledge, which organisations and individuals are invited to sign up to (https://www.kcl.ac.uk/research/obesity-pledge). The Nature Research journals have signed up to this pledge and Nature Reviews Endocrinology is proud to support the pledge and help end weight bias and obesity stigma. We pledge:

- To treat individuals with overweight and obesity with dignity and respect.

- To refrain from using stereotypical language, images and narratives that unfairly and inaccurately depict individuals with overweight and obesity as lazy, gluttonous and lacking willpower or self-discipline.

- To encourage and support educational initiatives aimed at eradicating weight bias through dissemination of modern-day knowledge of obesity and body-weight regulation.

- To encourage and support initiatives aimed at preventing weight-based discrimination in the workplace, education and health care settings ${ }^{1}$.

We feel strongly that everyone deserves to be treated with dignity and respect and that a person's body weight should not influence how they are treated in society. We hope that our readers will join us in supporting this pledge to end weight bias and obesity stigma, ensuring a fairer society for all.

1. Rubino, F. et al. Joint international consensus statement for ending stigma of obesity. Nat. Med. https://doi.org/10.1038/s41591-0200803-x (2020)

2. Blüher, M. Obesity: global epidemiology and pathogenesis Nat. Rev. Endocrinol. 15, 288-298 (2019).

3. Council of the Obesity Society. Obesity as a disease: the Obesity Society Council resolution. Obesity (Silver Spring) 16, 1151 (2008).

4. Spahlholz, J. Obesity and discrimination - a systematic review and meta-analysis of observational studies. Obes. Rev. 17, 43-55 (2016). 